ABSTRACT: The basis for cognitive impairment in Duchenne muscular dystrophy (DMD) is not well understood but may be related to abnormal expression of dystrophin in brain. The aim of this study was to determine whether regional brain glucose metabolism is altered in children with DMD and whether such metabolic disturbances are localized to regions shown to be normally rich in dystrophin expression. Ten boys (mean age, 11.8 years) with DMD and 17 normal adults as a control group (mean age, 27.6 years) underwent 2-deoxy-2 $\left[{ }^{18} \mathrm{~F}\right]$ fluoro-D-glucose positron emission tomography (PET) and neuropsychological evaluation. The PET data were analyzed by statistical parametric mapping (SPM). The SPM analysis showed five clusters of decreased glucose metabolism in children with DMD, including the medial temporal structures and cerebellum bilaterally and the sensorimotor and lateral temporal cortex on the right side. At the voxel level, significant glucose hypometabolism was found in the right postcentral and middle temporal gyri, uncus, and VIIIB cerebellar lobule, as well as in the left hippocampal gyrus and cerebellar lobule. The neuropsychological profile of the DMD group revealed borderline nonverbal intellectual functioning, impaired manual dexterity bilaterally, borderline cognitive functioning, and internalizing behavioral difficulties. Our findings demonstrate region-specific hypometabolism, as well as cognitive and behavioral deficits in DMD children. As the regions showing hypometabolism on PET include those normally rich in dystrophin expression, it will be important to determine whether the hypometabolic regions also show cytoarchitectural abnormalities related to the lack of dystrophin.

(C) 2002 Wiley Periodicals, Inc. Muscle Nerve 26: 506-512, 2002

\title{
ALTERED REGIONAL BRAIN GLUCOSE METABOLISM IN DUCHENNE MUSCULAR DYSTROPHY: A PET STUDY
}

\author{
JOON SOO LEE, MD, ${ }^{1}$ ZOLTÁN PFUND, MD, ${ }^{1}$ CSABA JUHÁSZ, MD, ${ }^{1}$ MICHAEL E. BEHEN, MA, ${ }^{1}$ OTTO MUZIK, PhD, ${ }^{1,3}$ \\ DIANE C. CHUGANI, PhD, ${ }^{1,3}$ MICHAEL A. NIGRO, DO, ${ }^{1,2}$ and HARRY T. CHUGANI, MD ${ }^{1-3}$ \\ ${ }^{1}$ Department of Pediatrics, Children's Hospital of Michigan, Wayne State University School of Medicine, 3901 Beaubien Blvd., Detroit, \\ Michigan 48201, USA \\ ${ }^{2}$ Department of Neurology, Children's Hospital of Michigan, Wayne State University School of Medicine, Detroit, Michigan, USA \\ ${ }^{3}$ Department of Radiology, Children's Hospital of Michigan, Wayne State University School of Medicine, Detroit, Michigan, USA
}

Accepted 25 June 2002

Duchenne muscular dystrophy (DMD) is an $\mathrm{X}$-linked recessive disorder characterized by the lack of dystrophin, the protein product of the DMD gene. ${ }^{43}$ Its overwhelming feature is progressive skeletal muscle weakness and degeneration resulting in

\footnotetext{
Abbreviations: CELF-III, Comprehensive Evaluation of Language Functions, Third Edition; DMD, Duchenne muscular dystrophy; FDG, 2-deoxy$2\left[{ }^{18} \mathrm{~F}\right]$ fluoro-D-glucose; FWHM, full-width-at-half-maximum; MRI, magnetic resonance imaging; MRS, magnetic resonance spectroscopy; PET, positron emission tomography; PIC-R, Personality Inventory for Children, Revised Edition; SPM, statistical parametric mapping; WISC-III, Weschler Intelligence Scales for Children, Third Edition; WRAML, Wide Range Assessment of Memory and Learning

Key words: brain glucose metabolism; Duchenne muscular dystrophy; neuropsychological assessment; positron emission tomography; statistical parametric mapping

Correspondence to: H.T. Chugani; e-mail: hchugani@pet.wayne.edu
}

(C) 2002 Wiley Periodicals, Inc. early death. ${ }^{31}$ Many patients with DMD show cognitive and behavioral impairments. ${ }^{3,18,25}$ Before discovery of dystrophin, these abnormalities were thought to be secondary effects of a chronic somatic disorder. It has been recognized, however, that although dystrophin is most abundant in skeletal muscle, significant levels are also found in cardiac and brain tissue. ${ }^{4,19}$ At least seven separate promoters at the DMD gene locus code for nervous system transcripts of distinct dystrophin isoforms. ${ }^{29}$ As dystrophin is not expressed in the brain of DMD patients, it has been hypothesized that this may be the basis for the central nervous system symptoms in DMD. 18,19,25

Although an early computed tomography study suggested mild cerebral atrophy in DMD patients, ${ }^{42}$ cranial magnetic resonance imaging (MRI) studies did not reveal any significant structural brain alterations in DMD subjects ${ }^{1}$ or in the $m d x$ mouse model 
of DMD. ${ }^{11}$ In contrast, functional neuroimaging studies have demonstrated a large variety of abnormalities in DMD and $m d x$ brain. Increased cerebellar choline/N-acetylaspartate ratios and higher than normal concentrations of choline compounds were found by ${ }^{1} \mathrm{H}$ magnetic resonance spectroscopy (MRS) in DMD patients ${ }^{34}$ and $m d x$ mice, ${ }^{40}$ suggesting gliosis or developmental abnormalities. Using ${ }^{31} \mathrm{P}$ MRS, increased ratios of inorganic phosphate to adenosine triphosphate and inorganic phosphate to phosphocreatine were reported in the cerebral cortex of DMD patients, suggesting possible mitochondrial impairment causing bioenergetic changes. ${ }^{41}$ Recently, decreased levels of free glucose were found associated with increased cellular flux of ${ }^{13} \mathrm{C}$ through pathways of oxidative glucose metabolism in $m d x$ mouse brain by MRS. ${ }^{33}$ A previous study of four older children with DMD using 2-deoxy-2 $\left[{ }^{18} \mathrm{~F}\right]$ fluoro-Dglucose (FDG) positron emission tomography (PET) demonstrated cerebellar glucose hypometabolism and variable involvement of cortical associative areas 3 ; however, a low-resolution PET scanner was used in that study, and group analysis was not performed. In addition, the calculated regional glucose metabolic values did not represent precisely the examined anatomical regions, as circular regions of interest were drawn for cortical areas, and not all brain regions were addressed.

In the present study, we examined children with DMD and adult control subjects using FDG PET to determine the differences in pattern of regional brain glucose metabolism. For an accurate and objective data analysis, we applied statistical parametric mapping (SPM). An additional goal of this study was to evaluate the neuropsychological data in children with DMD.

\section{MATERIALS AND METHODS}

Subjects. Ten boys (age range, 5.2-16.2 years; mean age, 11.8 years) with DMD were included in this study. All children had the typical features of DMD and elevated serum creatine kinase levels. Electromyography showed myopathic features and the diagnosis was confirmed by muscle biopsy in all children. All patients showed absent dystrophin immunoreactivity in muscle by Western blot analysis. DNA analysis was performed in seven patients and revealed deletions in the dystrophin gene in all of them.

All patients underwent MRI and FDG PET scans. The MRI studies produced normal findings. Eight patients underwent detailed neuropsychological evaluations involving assessment of global, verbal, and nonverbal intellectual functioning, receptive and expressive vocabulary, language processing, memory, attention, manual dexterity, and behavioral functioning. The battery included the following measures: Weschler Intelligence Scales for Children, Third Edition (WISC-III); Comprehensive Evaluation of Language Functions, Third Edition (CELFIII); Token Test; Wide Range Assessment of Memory and Learning (WRAML); Grooved Pegboard; and Personality Inventory for Children, Revised Edition (PIC-R). The psychometric properties of the above measures have been well established and the measures are widely used with both clinical and research populations. ${ }^{35}$ On nonverbal intellectual testing, administration procedures were adapted for patients whose motor functioning was impaired to a degree judged to adversely influence their ability to perform on timed tests of nonverbal abilities. Adaptations were those advocated by Sattler. ${ }^{35}$

To obtain normative neuroimaging data, we studied 17 normal adults (9 men and 8 women; age range, 21.1-38.2 years; mean age, 27.6 years). All control subjects had normal MRI and PET scans. None of the adults was taking any medication and none had a history of neurological or psychiatric disorder. Studies were performed in compliance with the regulations of Wayne State University $\mathrm{Hu}$ man Investigation Committee, and written informed consent of adult participants and parent or legal guardian of patients was obtained before all studies.

PET Scanning Procedure. Subjects fasted for $4 \mathrm{~h}$ prior to the PET studies. A venous line was established for injection of FDG $(0.143 \mathrm{mCi} / \mathrm{kg})$ produced using a CTI RDS-11 cyclotron (Knoxville, Tennessee). External stimuli were minimized by dimming the lights and discouraging interaction, so that studies reflected the resting awake state during the uptake period (0-30 min after injection). Sedation with intravenous pentobarbital or midazolam was used if necessary only after completion of the uptake period.

Scanning was performed using a CTI/Siemens EXACT/HR whole body positron tomograph. The scanner has a $15-\mathrm{cm}$ field of view and generates 47 image planes with a slice thickness of $3.125 \mathrm{~mm}$. The reconstructed image in-plane resolution obtained is $6.5 \pm 0.35 \mathrm{~mm}$ at full-width-at-half-maximum (FWHM) and $6.0 \pm 0.49 \mathrm{~mm}$ in the axial direction for the FDG PET (reconstruction parameters: Shepp-Logan filter with 1.1 cycles/cm cut-off frequency and Hanning filter with 0.20 cycles/pixel cut-off frequency). Calculated attenuation correction was performed as previously described. ${ }^{2}$ 
SPM Analysis. Although the SPM analysis has been validated only for children over 6 years of age, ${ }^{32}$ we included a 5.2-year-old patient in the analysis, as the orthogonal brain diameters (measured through the three Talairach reference lines [anterior commissure-posterior comissure, vertical and midline]) of this patient were not different from the same measures of the other nine patients who were above 6 years of age. To define objectively brain regions with abnormal glucose metabolism in the DMD patients, we assessed regionally specific differences between the patient group and the adult normal controls using linear contrasts. ${ }^{16}$

The PET volumes were processed using the SPM96 module (Wellcome Department of Cognitive Neurology, Institute of Neurology, London, UK) incorporated in the MEDx software package (Sensor Systems, Inc., Sterling, Virginia) installed on an SGI Octane workstation (Silicon Graphics, Inc., Mountain View, California). Spatial normalization into standard stereotactic space ${ }^{39}$ was performed using 12 affine parameters and a linear combination of threedimensional basis images to minimize the sum-ofsquares between an individual PET image volume and the PET image template. ${ }^{15}$ The basis images used were those that make up the lowest-frequency components of a three-dimensional discrete cosine transform. ${ }^{23}$ We used the default values of $4 \times 5 \times 4$ basis functions and applied eight iterations. The matrix size of the PET image template provided with the SPM96 software was $91 \times 109 \times 91$ voxels, with an isotropic voxel size of $2 \mathrm{~mm}^{3}$. In the process of spatial normalization, the PET image volumes were temporarily smoothed with a three-dimensional Gaussian kernel of $8 \mathrm{~mm}$ FWHM to match the resolution of the PET image template.

Following spatial normalization, all image volumes were smoothed with a three-dimensional Gaussian kernel of $12 \mathrm{~mm}$ FWHM. Our previous studies showed that this filter is adequate for the processing of pediatric brains. ${ }^{32}$ The confounding effect of global activity was removed using proportional scaling. A Student's unpaired $t$-test based on two contrasts (group 1 minus group 2, and group 2 minus group 1) was applied to the data in each group testing. The resulting $\operatorname{SPM}(\mathrm{t})$ was transformed to a normal distribution, $\mathrm{SPM}(\mathrm{Z})$, and thresholded at $P=0.002$ (uncorrected). Only those regions of more than 50 voxels attaining a corrected $P$ value of less than 0.05 were considered. The significance of each region was estimated using distributional approximations from the theory of Gaussian fields. ${ }^{16}$ The SPM(Z) maps were analyzed using two corrected levels of statistical inference: a voxel level (Z), and a cluster level $(\mathrm{k}, \mathrm{Z})$. The voxel level represents the probability (corrected for multiple comparisons based on the number of resolution elements in the image volume) of observing a Z-score of "Z" or higher, whereas the cluster level represents the probability of observing a cluster size " $\mathrm{k}$ " or larger with a maximal Z-score of "Z" or higher. The corrected significance level was chosen as $P<0.05$. The resulting significant differences in 3D image space were displayed collapsed into three orthogonal planes ("glass brain"). Regions of significant difference were overlaid on normalized T1-weighted images to facilitate correlation with anatomy. The Talairach coordinates ${ }^{39}$ are given in millimeters describing the location of significant voxels; $x$ defining the lateral displacement of this voxel from the midline (left $=$ negative), $y$ defining the anteroposterior position relative to the anterior commissure (posterior $=$ negative), and $z$ defining the vertical position relative to the line connecting the anterior and posterior commissure $($ down $=$ negative $)$.

Statistical Analysis. All statistical analyses were performed using the SPSS 10.0 statistical package (SPSS Inc., Chicago, Illinois). Associations between neuropsychological variables and age were analyzed via Pearson's product moment correlational analyses, corrected for multiple comparisons using a Bonferroni correction. Corrected $P<0.05$ was considered to be significant.

\section{RESULTS}

SPM Analysis. The analysis showed four clusters of decreased glucose metabolism in the children with DMD (Table 1), and one cluster with marginal significance. The clusters included the medial temporal structures and cerebellum bilaterally and the sensorimotor area and lateral temporal cortex on the right side (Table 1; Figs. 1-3). At the voxel level, significantly decreased glucose metabolism was found in the DMD group in the right postcentral and middle temporal gyri, uncus, and VIIIB cerebellar lobule, as well as in the left hippocampal gyrus and crus 1 cerebellar lobule (Table 1). In addition, there was a tendency for significance in the right hippocampus (Table 1). No significant increases were found in the DMD group as compared with the normal controls.

Neuropsychological Evaluation. Group means for neuropsychological evaluation of DMD children are presented in Table 2. The group neuropsychological profile revealed borderline global intellectual functioning, with low-average verbal intellectual and bor- 
Table 1. Results of SPM analysis: areas with decreased glucose metabolism in children with DMD.

\begin{tabular}{lccl}
\hline $\begin{array}{l}\text { Significant differences on cluster-level } \\
P \text {-value, }(k, Z)\end{array}$ & \multicolumn{2}{c}{ Maximal differences on the voxel level } \\
\cline { 2 - 4 } & $P$ value voxel level $(Z)$ & Talairach coordinate $x, y, z$ (mm) & Brain region (Brodmann's area) \\
\hline $0.007(386,5.42)$ & $0.000(5.42)$ & $60,-12,24$ & R postcentral gyrus (3) \\
$0.013(259,5.19)$ & $0.001(5.19)$ & $66,-42,-6$ & R middle temporal gyrus (21) \\
$0.030(227,4.81)$ & $0.008(4.81)$ & $-30,-12,-24$ & $L$ hippocampal gyrus (35) \\
$0.024(3067,4.71)$ & $0.012(4.71)$ & $28,-50,-50$ & R cerebellum, VIIIB \\
& $0.015(4.66)$ & $-28,-48,-42$ & $L$ cerebellum, crus (1) \\
$0.062(324,4.52)$ & $0.027(4.52)$ & $32,-14,-28$ & $R$ uncus (20) \\
& $0.080(4.24)$ & $28,-12,-16$ & R hippocampus (35) \\
\hline
\end{tabular}

$L$, left; $R$, right.

$k$ indicates the number of voxel elements included in the cluster.

derline nonverbal intellectual functioning. Expressive and receptive language fell in the average range, and visual memory functions were measured in the low-average range. Manual dexterity was impaired bilaterally. Measurement of behavioral functioning indicated borderline cognitive functioning and internalizing behavioral difficulties.

Correlational analyses between neuropsychological variables and age revealed no significant associations between age and any of the neuropsychological variables; however, trends in the direction of reduced manual dexterity for the dominant hand $(r=$ $-0.874 ; P=0.06)$ and receptive language $(r=$ $-0.937 ; P=0.072)$ were found in the older patients.
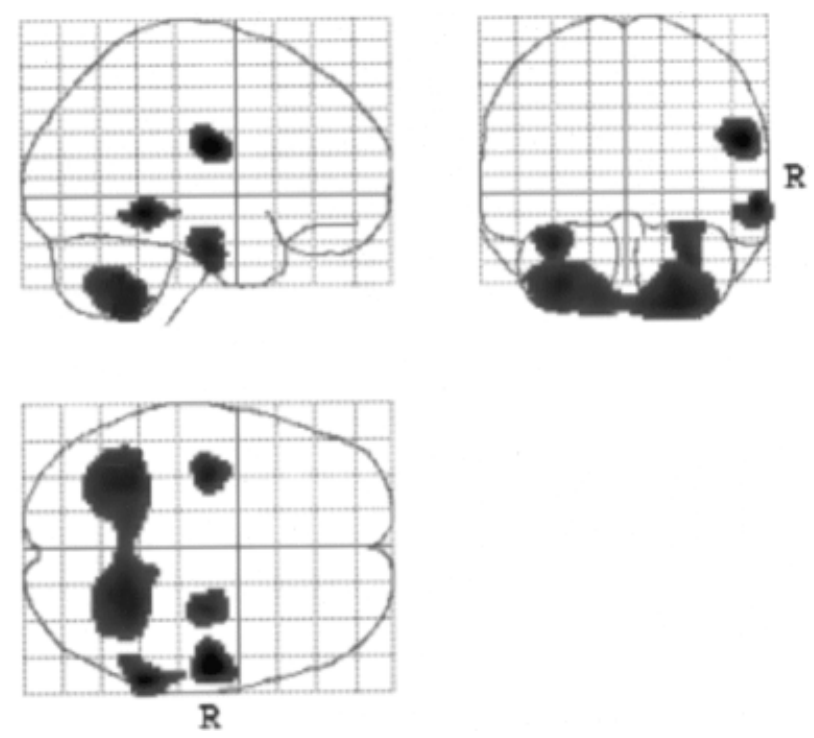

FIGURE 1. Composite figure summarizing SPM findings comparing children with DMD with adult controls. The results are displayed in a glass brain, with sagittal, coronal, and transverse views showing regions of significantly decreased tracer activity (with a corrected $P$ value $<0.05$ ). The figure demonstrates bilateral medial temporal and cerebellar decreases, as well as reduced activity in the right $(R)$ sensorimotor and lateral temporal areas in children with DMD.

\section{DISCUSSION}

In the present study, we determined a regional pattern of brain glucose metabolism in children with DMD using the SPM data-analysis method. The ideal control group for pediatric SPM analysis would be an age-matched group of normal children; however, this is not possible because of ethical restraints. The rationale for using an adult control group is based on our previous studies showing similar overall patterns of brain glucose metabolism in childhood $(>1$ year of age) and adulthood, despite huge differences in the absolute rates of glucose utilization. ${ }^{6,7} \mathrm{We}$ reasoned that if the regional pattern of glucose utilization is indeed fixed by 1 year of age, comparison of glucose PET scan using SPM, a technique that compares data that are normalized to the global mean, might be a useful approach for the study of pediatric disorders. Furthermore, SPM is used to compare patterns between groups and can be used to compare adult patterns with those of children older than 6 years of age. ${ }^{32}$ Previous studies using this analytic approach in different pediatric neuropsychiatric conditions $s^{5,24,32}$ provided considerably different results, and this supports the belief that the objective PET findings were related to the underlying disease rather than to developmental differences in regional brain glucose metabolism.

We found reduced regional brain glucose metabolism in children with DMD involving the sensorimotor area, temporal neocortex, medial temporal structures, and cerebellum. In normal brain, these areas are rich in dystrophin, which is restricted to postsynaptic membrane of neurons including neocortical pyramidal neurons (predominantly in deep frontal pyramidal layers), hippocampal CA regions, and the soma and dendritic regions of cerebellar Purkinje cells. ${ }^{21,26,27,29}$ In contrast, we did not find decreased glucose metabolism in subcortical gray matter structures and white matter where little or no dystrophin immunoreactivity has been identi- 
A
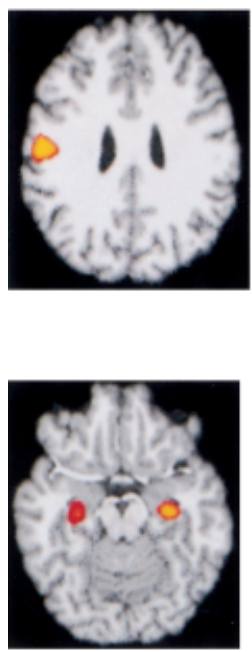

E
B
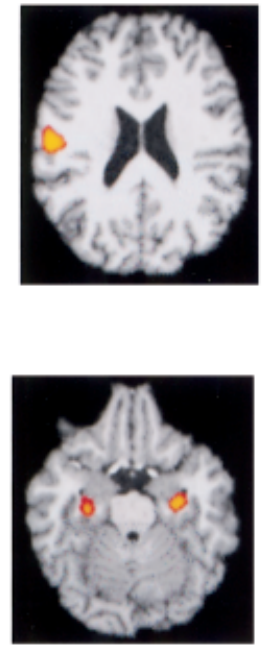

F
C
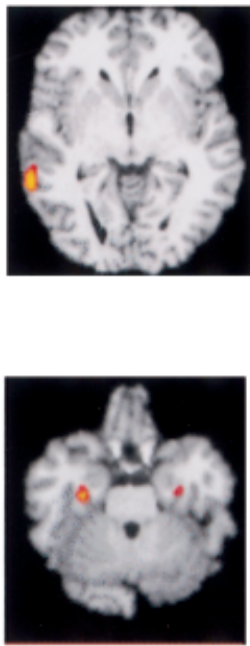

G
D
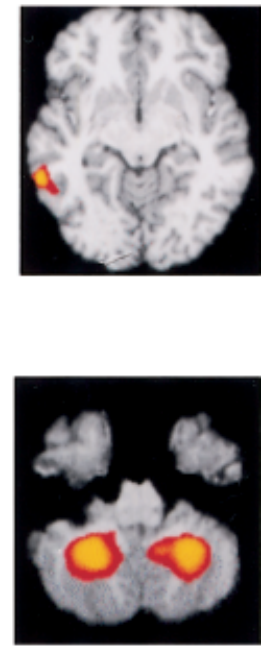

H

FIGURE 2. The SPM results of children with DMD versus adult control comparisons. The maps are superimposed on representative axial MR images in standardized space. Red and yellow areas in the right sensorimotor (A and B) and lateral temporal regions (C and D), as well as in the bilateral medial temporal structures $(\mathbf{E}-\mathbf{G})$ and cerebellum $(\mathbf{H})$ represent significant $(P<0.05)$ decreases of tracer activity in the DMD group. The left side of the images is the right side of the brain.

fied. ${ }^{26,27}$ This suggests an association between the pathological lack of dystrophin in regions normally abundant in dystrophin and presence of glucose hypometabolism in patients with DMD.

A

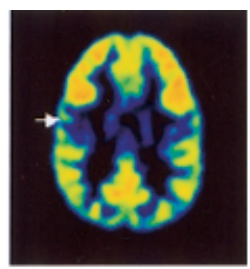

B

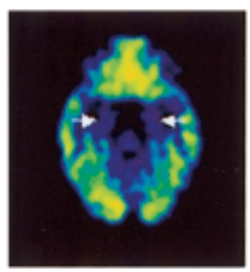

C
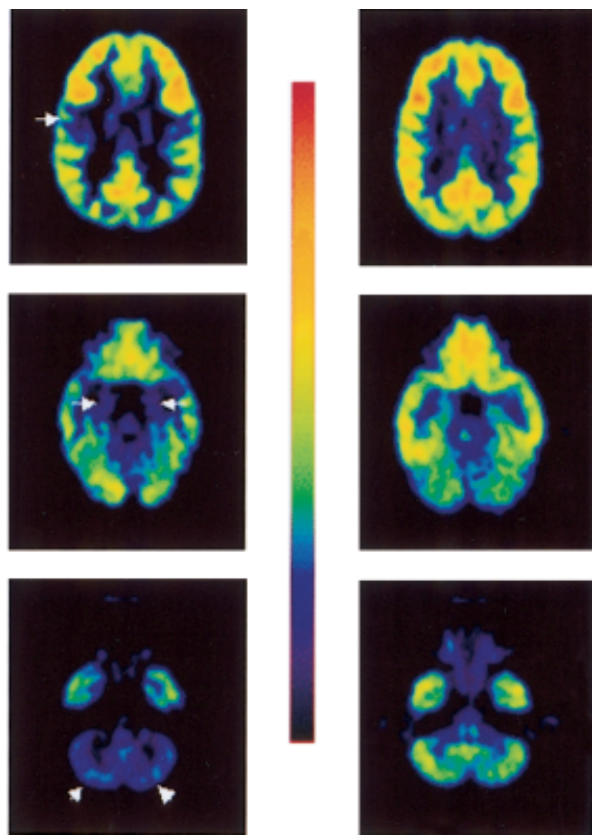

FIGURE 3. Transaxial FDG PET images in children with DMD (left column) and corresponding images in adult controls (right column). The tissue glucose metabolisms are color coded with red as maximum and black as minimum. The arrows show decreased glucose metabolism in the right sensorimotor cortex (A), bilateral medial temporal structures (B) and cerebellar cortex (C).
Our findings are partly consistent with the results of a previous FDG PET study on DMD subjects. ${ }^{3}$ That study also showed cerebellar hypometabolism in all examined patients and a variable involvement of the frontal, parietal, temporal, occipital, and cerebellar cortical areas; however, the results of that study were based on individual analyses, whereas our findings reflect group differences and show abnormalities in several additional regions (e.g., medial temporal structures), indicating common brain structures that are functionally abnormal in DMD. Still, individual variations of abnormal glucose metabolism likely occur and can be related to, for example, genetic heterogeneity of the disease. This issue is not addressed by the present study and would require PET analysis of a larger patient population with wellcharacterized genetic abnormalities.

Our findings indicate more decreases in glucose metabolism in the right hemisphere than in the left. It is possible that the asymmetric involvement may be related to functional asymmetries in the male brain, whereas the female brain appears to show more symmetry. For example, there is evidence that language functions are more lateralized in men than in women. ${ }^{38}$ Our findings in DMD patients suggesting increased susceptibility of the right hemisphere (in a disease of men) require further consideration and confirmation.

Our subjects with DMD showed individual variations in the distribution and extent of deficits on 


\begin{tabular}{|c|c|c|}
\hline Domain & Mean standard score ${ }^{\star}$ Mean T score ${ }^{\dagger}$ & Standard deviation (SD) \\
\hline \multicolumn{3}{|l|}{ Intellectual functioning (WISC-III) } \\
\hline Full Scale IQ & $79.0^{\star}$ & 9.0 \\
\hline Verbal IQ & $84.0^{*}$ & 12.0 \\
\hline Performance IQ & $78.0^{\star}$ & 9.0 \\
\hline \multicolumn{3}{|l|}{ Language } \\
\hline Expressive language (CELF-III) & $91.0^{*}$ & 12.0 \\
\hline Receptive language (Token Test) & $98.0^{*}$ & 11.0 \\
\hline \multicolumn{3}{|l|}{ Memory (WRAML) } \\
\hline Memory screen index & $88.0^{\star}$ & 8.0 \\
\hline \multicolumn{3}{|l|}{ Manual dexterity (Grooved Pegboard) } \\
\hline Dominant hand & $56.0^{\star}$ & 16.0 \\
\hline Nondominant hand & $46.0^{*}$ & 12.0 \\
\hline \multicolumn{3}{|l|}{ Behavior (PIC-R) } \\
\hline Factor I & $52.8^{\dagger}$ & 11.1 \\
\hline Factor II & $61.8^{\dagger}$ & 6.4 \\
\hline Factor III & $67.3^{\dagger}$ & 17.7 \\
\hline Factor IV & $68.2^{\dagger}$ & 15.4 \\
\hline
\end{tabular}

*Standard score (normal mean = 100; SD = 15); higher score represents better performance.

${ }^{t} T$ score (normal mean = 50; SD = 10); higher score represents greater behavior problem.

neuropsychological measures, consistent with previous reports in patients with DMD who showed various degrees of intellectual impairment, cognitive deficits, and behavioral abnormalities. ${ }^{3,13,18,25,34,41} \mathrm{~A}$ close relationship was found previously between the loss of Dp140 and Dp71 brain dystrophin isoforms and intellectual impairment in DMD, suggesting that the cognitive deficits are very likely the consequences of altered synaptic plasticity, neuronal dysfunction, and cell damage in dystrophin-absent DMD brain tissue. ${ }^{3,13,29,30}$ The pattern of glucose hypometabolism detected in our study may suggest links between dysfunction of sensorimotor areas and reduced manual dexterity, ${ }^{8,28}$ temporal cortical areas and cognitive and behavioral impairment, ${ }^{9,12}$ as well as cerebellum and intellectual and cognitive deficit. ${ }^{14,37}$

The precise function of dystrophin in cerebral and cerebellar cortical cells and its relation to glucose uptake and metabolism has yet to be elucidated. Dystrophin may transduce molecular signals from the extracellular environment to cell nucleus ${ }^{29}$; however, it is not known how the impaired signal transduction can lead to abnormal intracellular glucose uptake and metabolism. In neuropathological studies of DMD and $m d x$ mouse there is evidence of neuronal loss and gliosis, as well as abnormal arborization, reduced dendritic length, and altered shape of pyramidal cells. ${ }^{22,36}$ Whether these abnormalities occur during early development as a consequence of aberrant dystrophin expression or are progressive in nature as the disease advances is not clear. It should be pointed out that neither the present nor previous studies ${ }^{3,25}$ revealed progressive mental retardation or other progressive central nervous system symptoms in DMD, which might be expected if there is steady cell damage after birth due to membrane instability that resulted in altered intracellular ion homeostasis. ${ }^{10,17,20}$ Thus, the abnormalities detected by FDG PET may reflect local cytoarchitectural abnormalities associated with altered neural development, as dystrophin may regulate distinct aspects of neurogenesis, neuronal migration, and cellular differentiation. ${ }^{29}$ It will be important, in our subjects, to obtain longitudinal PET data to determine whether hypometabolism is progressive. Finally, postmortem neuropathological correlations with regional cerebral glucose metabolism may further elucidate whether PET studies can localize dystrophin-deficient areas of brain injury in DMD.

The authors thank Thomas J. Mangner, PhD, and Pulak Chakraborty, PhD, for the reliable synthesis of the PET tracer. They also thank Galina Rabkin, Teresa Jones, Giselle Baillargeon, Crystal Baird, and Peter Ellepola for their expert technical assistance in performing the PET studies.

\section{REFERENCES}

1. Al-Qudah AA, Kobayashi J, Chuang S, Dennis M, Ray P. Etiology of intellectual impairment in Duchenne muscular dystrophy. Pediatr Neurol 1990;6:57-59.

2. Bergström M, Litton J, Eriksson L, Bohm C, Blomqvist G. Determination of object contour from projections for attenuation correction in cranial positron emission tomography. J Comput Assist Tomogr 1982;6365-6372.

3. Bresolin N, Castelli E, Comi GP, Felisari G, Bardoni A, Perani D, Grassi F, Turconi A, Mazzucchelli F, Gallotti D, Moggio M, 
Prelle A, Ausenda C, Fazio G, Scarlato G. Cognitive impairment in Duchenne muscular dystrophy. Neuromuscul Disord 1994;4:359-369.

4. Chamberlain JS, Pearlman JA, Muzny DM, Gibbs RA, Ranier JE, Caskey CT, Reeves AA. Expression of the murine Duchenne muscular dystrophy gene in muscle and brain. Science 1988;239:1416-1418.

5. Chugani HT, Behen ME, Muzik O, Juhász C, Nagy F, Chugani DC. Local brain functional activity following early deprivation: a study of post-institutionalized Romanian orphans. Neuroimage 2001;14:1290-301.

6. Chugani HT, Phelps ME. Maturational changes in cerebral function in infants determined by ${ }^{18}$ FDG positron emission tomography. Science 1986;231:840-843.

7. Chugani HT, Phelps ME, Mazziotta JC. Positron emission tomography study of human brain functional development. Ann Neurol 1987;22:487-497.

8. Darian-Smith I, Galea MP, Darian-Smith C. Manual dexterity: how does the cerebral cortex contribute? Clin Exp Pharmacol Physiol 1996;23:948-956.

9. Devinsky O, Bear D. Varieties of aggressive behavior in temporal lobe epilepsy. Am J Psychiatry 1984;141:651-656.

10. Dunn JF, Burton KA, Dauncey MJ. Quabain sensitive $\mathrm{Na}^{+} /$ $\mathrm{K}\left({ }^{+}\right)$-ATPase content is elevated in $m d x$ mice: implications for the regulation of ions in dystrophic muscle. J Neurol Sci 1995;133:11-15.

11. Dunn JF, Zaim-Wadghiri Y. Quantitative magnetic resonance imaging of the mdx mouse model of Duchenne muscular dystrophy. Muscle Nerve 1999;22:1367-1371.

12. Eichenbaum H. A cortical-hippocampal system for declarative memory. Nat Rev Neurosci 2000;1:41-50.

13. Felisari G, Martinelli Boneschi F, Bardoni A, Sironi M, Comi GP, Robotti M, Turconi AC, Lai M, Corrao G, Bresolin N. Loss of Dp140 dystrophin isoform and intellectual impairment in Duchenne dystrophy. Neurology 2000;55:559-564.

14. Fiez JA, Petersen SE, Cheney MK, Raichle ME. Impaired non-motor learning and error detection associated with cerebellar damage. A single case study. Brain 1992;115:155-178.

15. Friston KJ, Ashburner J, Poline JB, Frith CD, Heather JD, Frackowiak RSJ. Spatial registration and normalization of images. Hum Brain Mapping 1995;2:165-189.

16. Friston KJ, Holmes AP, Worsley KJ, Poline JB, Frith CD, Frackowiak RSJ. Statistical parametric maps in functional imaging: a general approach. Hum Brain Mapping 1995;2:189210.

17. Haws CM, Lansman JB. Calcium-permeable ion channels in cerebellar neurons from $m d x$ mice. Proc R Soc Lond B Biol Sci 1991;244:185-189.

18. Hinton VJ, De Vivo DC, Nereo NE, Goldstein E, Stern Y. Poor verbal working memory across intellectual level in boys with Duchenne dystrophy. Neurology 2000;54:2127-2132.

19. Hoffman EP, Brown RH Jr, Kunkel LM. Dystrophin: the protein product of the Duchenne muscular dystrophy locus. Cell 1987;51:919-928.

20. Hopf FW, Steinhardt RA. Regulation of intracellular free calcium in normal and dystrophic mouse cerebellar neurons. Brain Res 1992;578:49-54.

21. Huard J, Tremblay JP. Localization of dystrophin in the Purkinje cells of normal mice. Neurosci Lett 1992;137:105-108.

22. Jagadha V, Becker LE. Brain morphology in Duchenne muscular dystrophy: a Golgi study. Pediatr Neurol 1988;4:87-92.

23. Jain AK. Fundamentals of digital image processing. London: Prentice-Hall; 1998. 592 p.
24. Juhász C, Behen ME, Muzik O, Chugani DC, Chugani HT. Bilateral prefrontal and temporal neocortical hypometabolism in children with epilepsy and aggression. Epilepsia 2001; 42:991-1001

25. Karagan NJ. Intellectual functioning in Duchenne muscular dystrophy: a review. Psychol Bull 1979;86:250-259.

26. Lidov HG, Byers TJ, Kunkel LM. The distribution of dystrophin in the murine central nervous system: an immunocytochemical study. Neuroscience 1993;54:167-187.

27. Lidov HG, Byers TJ, Watkins SC, Kunkel LM. Localization of dystrophin to postsynaptic regions of central nervous system cortical neurons. Nature 1990;725-728.

28. Liu Y, Rouiller EM. Mechanisms of recovery of dexterity following unilateral lesion of the sensorimotor cortex in adult monkeys. Exp Brain Res 1999;128:149-159.

29. Mehler MF. Brain dystrophin, neurogenetics and mental retardation. Brain Res Brain Res Rev 2000; 32:277-307.

30. Moizard MP, Toutain A, Fournier D, Berret F, Raynoud M, Billard C, Andres C, Moraine C. Severe cognitive impairment in DMD: obvious clinical indication for Dp71 isoform point mutation. Eur J Hum Genet 2000;552-556.

31. Moser H. Duchenne muscular dystrophy: pathogenetic aspects and genetic prevention. Hum Genet 1984;66:17-40.

32. Muzik O, Chugani DC, Juhász C, Shen C, Chugani HT. Statistical parametric mapping: assessment of application in children. Neuroimage 2000;12:538-549.

33. Rae C, Griffin JL, Blair DH, Bothwell JH, Bubb WA, Maitland A, Head S. Abnormalities in brain biochemistry associated with lack of dystrophin: studies of the $m d x$ mouse. Neuromuscul Disord 2002;12:121-129.

34. Rae C, Scott B, Thompson CH, Dixon RM, Dumughn I, Kemp GJ, Male A, Pike M, Styles P, Radda GK. Brain biochemistry in Duchenne muscular dystrophy: a $1 \mathrm{H}$ magnetic resonance and neuropsychological study. J Neurol Sci 1998;160:148-157.

35. Sattler JM. Assessment of children, 3rd edition. San Diego: Sattler Publisher; 1992. p 96-137.

36. Sbriccoli A, Santarelli M, Carretta D, Pinto F, Granato A, Minciacchi D. Architectural changes of the cortico-spinal system in the dystrophin defective mdx mouse. Neurosci Lett 1995;200:53-56.

37. Schmahmann JD. An emerging concept. The cerebellar contribution to higher function. Arch Neurol 1991;48:1178-1187.

38. Shaywitz BA, Shaywitz SE, Pugh KR, Constable RT, Skudlarski P, Fulbright RK, Bronen RA, Fletcher JM, Shankweiler DP, Katz L, Gore JC, Herbert Y. Sex differences in the functional organization of the brain for language. Nature 1995;373:607609.

39. Talairach J, Tournoux P. Co-planar stereotaxic atlas of the human brain. Stuttgart: Thieme; 1988. 12 p.

40. Tracey I, Dunn JF, Parkes HG, Radda GK. An in vivo and vitro 1H-magnetic resonance spectroscopy study of $\mathrm{mdx}$ mouse brain: abnormal development or neural necrosis? J Neurol Sci 1996;141:13-18.

41. Tracey I, Scott RB Thompson CH, Dunn JF, Barnes PR, Styles P, Kemp GJ, Rae CD, Pike M, Radda GK. Brain abnormalities in Duchenne muscular dystrophy: phosphorus-31 magnetic resonance spectroscopy and neuropsychological study. Lancet 1995;345:1260-1264.

42. Yoshioka M, Okuno T, Honda Y, Nakano Y. Central nervous system involvement in progressive muscular dystrophy. Arch Dis Child 1980;55:589-594.

43. Zubrzycka-Gaarn EE, Bulman DE, Karpati G, Burghes AH, Belfall B, Klamut HJ, Talbot J, Hodges RS, Ray PN, Worton RG. The Duchenne muscular dystrophy gene product is localized in sarcolemma of human skeletal muscle. Nature $1988 ; 333 ; 466-469$. 